\title{
A Common-Ground Review of the Potential for Machine Learning Approaches in Electrocardiographic Imaging Based on Probabilistic Graphical Models
}

\author{
Jaume Coll-Font ${ }^{1}$, Linwei Wang ${ }^{2}$, Dana H Brooks ${ }^{3}$ \\ ${ }^{1}$ Computational Radiology Lab, Children's Hospital, Boston (MA), USA \\ ${ }^{2}$ Rochester Institute of Technology, Rochester (NY), USA \\ ${ }^{3}$ SPIRAL Group, ECE Dept. Northeastern University, Boston (MA), USA
}

\begin{abstract}
Machine learning (ML) methods have seen an explosion in their development and application. They are increasingly being used in many different fields with considerable success. However, although the interest is growing, their impact in the field of electrocardiographic imaging (ECGI) remains limited. One of the main reasons that $M L$ has yet to become more prevalent in ECGI is that the published literature is scattered and there is no common ground description and comparison of these methods in an ML framework.

Here we address this limitation with a review of ECGI methods from the perspective of $M L$. We will use probabilistic modeling to provide a common ground framework to compare different methods and well known approaches. Finally, we will discuss which approaches have been used to do inference on these models and which alternatives could be utilized as the methods in ML become more mature.
\end{abstract}

\section{Introduction}

Electrocardiographic Imaging (ECGI) attempts to image the electrical activity in the heart from the electrical recordings on the body surface and a model of their relationship. This is a challenging problem due to the ill-posedness of the inverse problem being solved [1] and, thus, all methods have to introduce prior knowledge as assumptions about the solution, the noise and/or the model to help stabilize the inverse. A classical method to solve this problem and the reference for most approaches is Tikhonov regularization [2], which solves Equation 1].

$$
\min _{x}\|y-A x\|_{2}^{2}+\lambda\|x\|_{2}^{2}
$$

where $x$ is a vector of heart surface potentials at a chosen set of nodes, $y$ is a corresponding vector of body surface potentials, and the matrix $A$ encodes a solution to the forward problem that relates $x$ to $y$. For simplicity here we assume that are are only considering a single time sample here.

The balance between the residual $\|y-A x\|_{2}^{2}$ and the regularization term $\lambda\|x\|_{2}^{2}$-which enforces the assumed prior knowledge - in Equation 1 is typical for inverse solvers. Other regularization approaches modify the regularization term, introduce hard constraints to the objective function or project the solutions onto a "valid" set.

The field of Machine Learning (ML) has seen an explosion in the number of publications and corresponding technical development and are increasingly being used in many fields with considerable success. In particular, Bayesian ML approaches allow us to model the data through the dependence relationships between random variables (RVs) and their probability distributions. These tools could help researchers in ECGI to properly model their assumptions about the problem and easily test them. However, although the interest is growing, the impact of ML in the field remains limited. One of the main reasons that ML has yet to become more prevalent in ECGI is that the published literature is scattered and there is no common ground description and comparison of these methods.

Here, we describe both classic and newer ECGI methods through the lens of ML. We use the parallel between classical optimization methods and Maximum-APosteriori (MAP) inference to derive the assumed probabilistic models in the ECGI methods. This framework will elucidate the inherent modeling assumptions, allow for comparison across methods, and easily incorporate different types of assumed prior knowledge. Finally, we will provide a quick glance at some inference approaches that can be applied to the models described ${ }^{1}$

\footnotetext{
${ }^{1}$ We have tried to include a representative and broad sample of published methods but have by necessity (due to both space limitations and our authorial limitations) omitted or missed some relevant methods; we apologize to those investigators and encourage them to contact us with any concerns.
} 


\section{Theoretical background}

The probabilistic model describing the most basic ECGI method is the following:

$$
\begin{aligned}
p(x) & =\mathcal{N}(x \mid 0, \rho) \\
p(y \mid x) & =\mathcal{N}(A x \mid \sigma),
\end{aligned}
$$

where $\mathcal{N}(\cdot)$ is a Gaussian distribution and $\sigma$ and $\rho$ are known scalar variances. The objective function in Equation 1 is then equivalent to doing inference on this model with a maximum a posteriori (MAP) objective, with $\lambda=$ $\frac{\sigma}{\rho}$. This is a very simple probabilistic model as it only includes the observed data $y$ and the latent variable $x$ and assumes known variances and forward matrix. However, it is the building block from which we will develop the rest of models we describe. We will do so by adding new assumptions about the relevant RVs as well as introducing assumed prior physiological knowledge about them.

\section{Modeling Approaches}

Even though the potentials on the heart have a strong spatial structure, Tikhonov regularization does not assume any spatial correlation across the heart. Exploiting this characteristic has been the common theme of many new ECGI methods in the literature. The simplest models are the higher order versions of Tikhonov, which introduce a fixed correlation structure between nearby nodes through a regularization matrix $R$. In those models, the prior used to describe the potential distribution is a multivariate Gaussian with covariance matrix given by $\Sigma=R^{-1}$. Tikhonov $2^{\text {nd }}$ order uses the Laplacian operator of the heart geometry to encourage higher correlation between neighboring nodes on the heart geometry [2, 3]. Changing the covariance matrix modifies the type of "ground truth" being enforced. For example, some groups have modified the Laplacian operator to enforce correlation between nodes on both sides of the myocardial wall [4-6] or introduced varying weights on the covariance matrix to favor more uncertainty in nodes on the epicardium than on the endocardium [7]. The previous methods assume known covariance matrices -i.e. regularization matrices- however, these can also be learned from the data. The covariance parameters can be treated as RVs and thus be learned jointly with the unknown heart potentials [8,9] or derived a priori from simulations or ex-vivo recordings [10].

Spatial modeling is not limited only to employing different covariance matrices with a Gaussian distribution. It is also possible to change the base probability distribution

\footnotetext{
${ }^{2}$ Note that many regularization matrices are not full rank. In those cases, the corresponding prior distribution is degenerate and is ill-defined for some dimensions. To provide stability, it is then necessary to increase the rank, by introducing a small weight to the diagonal of the covariance matrix to stabilize the results.
}

used. For example, it is possible to use the Laplace distribution, which is equivalent to an L1 norm constraint of the solution and used to favor the presence of a small number of large values. Total Variation uses this same distribution -with a covariance matrix equal to the inverse gradient operator - to allow for a small number of large gradients on the heart potentials [11-15]. Other groups have extended this idea to induce sparsity with generalized Gaussian distributions - equivalent to the Lp norm in classical optimization [16, 17].

The heart potentials also have a strong temporal structure that is often leveraged to add prior knowledge. The simplest approach is to extend the dimensions of the latent variable $x$ to include time and define the covariance matrix to introduce temporal structure. For example, stacking the time instances on top of each other into a block vector and employing a spatio-temporal covariance matrix with a block diagonal form assumes independence between time instances. Any non-zero entry outside of the main block diagonal introduces correlation in time. For example, if two regularization matrices $R$ and $T$-in space and timeare used, the inverse covariance has the spatial regularization $R$ repeated along the diagonal and the temporal regularization $T$ repeating in off-diagonal positions [18]. Greensite and Huiskamp [19] introduced an optimal structure based on assumptions of separability of temporal and spatial correlations; for a comparative study of three such methods see [20]. However, the most common approach to model temporal behavior is to use deterministic restrictions of the solution space. This is done via projection of the data and solutions onto a feasible set [4, 21-[25] or through explicit constraints in the optimization [26]. These temporal restrictions correspond to setting probability zero to the unfeasible space and creating truncated probability distributions.

An even more restrictive approach is to assume a specific temporal shape for the potentials. Activation-based methods do this, solving for unknown activation times on each node of the heart [27]. This restriction can be refined with more complex propagation models for the heart potentials. These models are equivalent to Hidden Markov probabilistic models, typically of order $1[28]$. The complexity of the model can vary from a simple curve defining the wave-front [29] to a full propagation models [12].

The models described so far only introduce prior knowledge about the heart potentials. It is possible, however, to also characterize the uncertainty introduced by other parameters. For example, a set of parameters that is often assumed fixed but that also introduces uncertainty is contained in the forward solutions. Miss-specifications of the geometry such as position of the heart or conductivities of the organs introduce error into the forward model and thus the inverse solutions. The literature addressing this prob- 
lem is still small, but there has been some work trying to characterize the uncertainty introduced by these model errors [30-32] and, more recently to correct for those from the data [33. 35].

Other parameters that can be further characterized as an $\mathrm{RV}$ are those that describe properties of the physiological propagation models. These models invariably depend on underlying parameters, typically chosen from "healthy" priors, post-mortem hearts or animal models. This RV can be set a priori [36, 37], learned outisde of the inverse solution with discriminative methods or learned jointly with the heart potentials [38-40].

It is important to note that the extension of these models with new variables increases the complexity of the model. Deep probabilistic models usually demand more resources to compute and, more importantly, more data for inference. The curse of dimensionality limits the number of parameters added and they should only be introduced if enough prior knowledge exists about them to learn them correctly with reasonable probability.

\section{Inference}

So far we have used the equivalence between classical optimization and MAP inference to develop a probabilistic framework for ECGI. A key advantage of translating standard classical optimization problems to probabilistic models is that it is then possible to test different inference methods from the ML literature. Variational Inference (VI), Markov Chain Monte Carlo (MCMC) methods or, more recently, disentanglement approaches in the deep learning literature, all provide estimates of the full posterior distribution and not just its mode or mean. Thus, one can include an estimate of the uncertainty of the results [12, 28, 31, 40, 42].

One significant limitation of these methods is that they require advanced knowledge in ML to design and code. Fortunately, there has been a considerable recent effort in ML research to develop probabilistic programming languages that offer readily usable black-box variational inference and MCMC algorithms [43]. With these tools, it is possible to describe the probabilistic model directly in a tailored programming language and then learn the posterior distributions without the limitation of having to develop the entire inference structure.

\section{Conclusions}

We have described some relevant methods in the ECGI literature in the context of probabilistic modeling. This framework helps contextualize efforts from different groups and may allow further development with incorporation of new prior knowledge about the problem. Finally, we have suggested how some inference techniques in the ML literature may facilitate the adoption of advanced ML methods in ECGI.

\section{References}

[1] Pullan A.J. et.al. The Inverse Problem of Electrocardiography. In Comp. Elec. Springer. ISBN 9781848820456, 2010; 1-51.

[2] Milanič M. et.al. Assessment of Regularization Techniques for Electrocardiographic Imaging. J Electrocardiol 2014; 47(1):20-8. ISSN 1532-8430.

[3] Wang Y, Rudy Y. Application of the Method of Fundamental Solutions to Potential-Based Inverse Electrocardiography. Ann Biomed Eng aug 2006;34(8):1272-1288. ISSN 0090-6964.

[4] Erem B. et.al. Using Transmural Regularization and Dynamic Modeling for Noninvasive Cardiac Potential Imaging of Endocardial Pacing with Imprecise Thoracic Geometry. IEEE TMI mar 2014;33(3):726-738. ISSN 02780062.

[5] Schuler S. et.al. ECG Imaging of Simulated Atrial Fibrillation: Imposing Epi-Endocardial Similarity Facilitates the Reconstruction of Transmembrane Voltages. In CinC, volume 44. ISSN 2325887X, 2017; 1-4.

[6] Liu Z. et.al. Noninvasive Reconstruction of ThreeDimensional Ventricular Activation Sequence from the Inverse Solution of Distributed Equivalent Current Density. IEEE TMI oct 2006;25(10):1307-1318. ISSN 0278-0062.

[7] Potyagaylo D. et.al. Local Regularization of Endocardial and Epicardial Surfaces for Better Localization of Ectopic Beats in the Inverse Problem of ECG. CinC 2014;837-840. ISSN 2325-8861.

[8] Kaipo J, Somersalo E. Statistical and Computational Inverse Problems. Springer, 2004. ISBN 0387220739.

[9] Serinagaoglu Y. et.al. Bayesian Solutions and Performance Analysis in Bioelectric Inverse Problems. IEEE Transactions on Biomedical Engineering jun 2005;52(6):10091020. ISSN 0018-9294.

[10] Cluitmans M.J.M. et.al. Physiology-Based Regularization of the Electrocardiographic Inverse Problem. Med Biol Eng Comput 2017;55(8):1353-1365. ISSN 17410444.

[11] Xu J. et.al. Noninvasive Transmural Electrophysiological Imaging Based on Minimization of Total-Variation Functional. IEEE TMI may 2014;33(9):1-15. ISSN 1558-254X.

[12] Xu J. et.al. Robust Transmural Electrophysiological Imaging : Integrating Sparse and Dynamic Physiological Models into ECG-based Inference. In MICCAI. 2015; .

[13] Coll-Font J. et.al. Evaluation of 2-norm Versus Sparsity Regularization in Spline-based Joint Reconstruction of Epicardial and Endocardial Potentials from Body-surface Measurements. In CinC, volume 42. IEEE. ISBN 9781509006854. ISSN 2325887X, 2015; 957-960.

[14] Wang D. et.al. Inverse Electrocardiographic Source Localization of Ischemia: An Optimization Framework and Finite Element Solution. J Comput Phys oct 2013;250:403424. ISSN 0021-9991.

[15] Coll-font J. et.al. A Statistical Approach to Incorporate Multiple ECG or EEG Recordings with Artifactual Variability into Inverse Solutions. In ISBI. Brooklyn: IEEE, 2015; 1053-1056. 
[16] Rahimi A. et.al. Electrophysiological Imaging of Volumetric Infract Border Using a Spatio-Temporal Lp-Norm Constraint. In ISBI. 2014; 612-615.

[17] Ghimire S, Wang L. L0 Norm Based Sparse Regularization for Non-invasive Infarct Detection using ECG Signal. In Computing in Cardiology (CinC), volume 44. 2017; 1-4.

[18] Brooks D.H. et.al. Inverse Electrocardiography by Simultaneous Imposition of Multiple Constraints. IEEE TBME jan 1999;46(1):3-18. ISSN 0018-9294.

[19] Greensite F, Huiskamp G. An improved method for estimating epicardial potentials from the body surface. IEEE Trans Biomed Eng 1998;45:98-104.

[20] Zhang Y, Ghodrati A, Brooks DH. An analytical comparison of three spatio-temporal regularization methods for dynamic linear inverse problems in a common statistical framework. Inverse Problems 2005;21:357-382.

[21] Huiskamp G, Greensite F. A New Method for Myocardial Activation Imaging. IEEE TBME jun 1997;44(6):433-46. ISSN 0018-9294.

[22] Guillem M. et.al. Noninvasive Localization of Maximal Frequency Sites of Atrial Fibrillation by Body Surface Potential Mapping. Circ Arrhythmia Elec 2013;6(2):294-301. ISSN 19413149.

[23] Coll-Font J. et.al. A Potential-Based Inverse Spectral Method to Noninvasively Localize Discordant Distributions of Alternans on the Heart from the ECG. IEEE TBME 2018;65(7):1554-1563. ISSN 15582531.

[24] Schuler S. et.al. Electrocardiographic Imaging Using a Spatio-Temporal Basis of Body Surface PotentialsApplication to Atrial Ectopic Activity. Frontiers in Physiology 2018;9(August):1-13. ISSN 1664-042X.

[25] Cluitmans M.J.M. et.al. Wavelet-Promoted Sparsity for Non-Invasive Reconstruction of Electrical Activity of the Heart. Med Biol Eng Comput 2018;1-12. ISSN 17410444.

[26] Messnarz B. et.al. A New Spatiotemporal Regularization Approach for Reconstruction of Cardiac Transmembrane Potential Patterns. IEEE TBME feb 2004;51(2):273-81. ISSN 0018-9294.

[27] van Dam P.M. et.al. Quantitative Localization of Premature Ventricular Contractions Using Myocardial Activation ECGI from the Standard 12-lead Electrocardiogram. J Electrocardiol sep 2013;46(6):574-579. ISSN 1532-8430.

[28] Bishop C. Pattern Recognition and Machine Learning (Information Science and Statistics). Secaucus, NJ, USA: Springer-Verlag New York, Inc., 2006. ISBN 0387310738.

[29] Ghodrati A. et.al. Wavefront-based Models for Inverse Electrocardiography. IEEE TBME sep 2006;53(9):182131. ISSN 0018-9294.

[30] Weber F.M. et.al. Predicting Tissue Conductivity Influences on Body Surface Potentials: an Efficient Approach Based on Principal Component Analysis. IEEE TBME feb 2011; 58(2):265-73. ISSN 1558-2531.

[31] Swenson D.J. et.al. Cardiac Position Sensitivity Study in the Electrocardiographic Forward Problem Using Stochastic Collocation and Boundary Element Methods. Ann Biomed Eng Dec 2011;39(12):2900-10. ISSN 00906964.

[32] Rahimi A. et.al. Toward Clinically-Feasible Noninvasive
Electrophysiological Imaging : Investigating the Impact of Local Anatomical Details. In STACOM. 2011; 188-197.

[33] Rodrigo M. et.al. Solving Inaccuracies in Anatomical Models for Electrocardiographic Inverse Problem Resolution by Maximizing Reconstruction Quality. IEEE TMI 2017; 25:1-1. ISSN 0278-0062.

[34] Coll-Font J. et.al. ECG-based Reconstruction of Heart Position and Orientation with Bayesian Optimization. In CinC, volume 44. ISSN 2325887X, 2017; .

[35] Potyagaylo D. et.al. Influence of Modeling Errors on the Initial Estimate for Nonlinear Myocardial Activation Times Imaging Calculated with Fastest Route Algorithm. IEEE Transactions on Biomedical Engineering 2016;9294(c):11. ISSN 0018-9294.

[36] Giffard-Roisin S. et.al. Non-Invasive Personalisation of a Cardiac Electrophysiology Model from Body Surface Potential Mapping. IEEE TBME 2016;64(9):1-1. ISSN 00189294.

[37] He B. et.al. Noninvasive Imaging of Cardiac Transmembrane Potentials Within Three-Dimensional Myocardium by Means of a Realistic Geometry Anisotropic Heart Model. IEEE TBME 2003;50(10):1190-1202. ISSN 00189294.

[38] Dhamala J. et.al. Spatially-Adaptive Multi-Scale Optimization for Local Parameter Estimation in Cardiac Electrophysiology. IEEE TMI 2017;36(9):1966-1978.

[39] Ghimire S. et.al. A Variational Approach to Sparse Model Error Estimation in Cardiac Electrophysiological Imaging. In MICCAI, volume 1. ISBN 978-3-319-66184-1. ISSN 978-3-319-66181-0, 2017; 745-753.

[40] Dhamala J. et.al. Quantifying the Uuncertainty in Model Parameters Using Gaussian Process-based Markov Chain Monte Carlo in Cardiac Electrophysiology. Med Image Anal 2018;48:43-57. ISSN 13618423.

[41] Siddharth N. et.al. Learning Disentangled Representation in Deep Generative Models. In ICLR. 2017; 1-12.

[42] Rahimi A. Uncertainty Quanti cation and Reduction in Cardiac Electrophysiological Imaging Uncertainty Quantification and Reduction in Cardiac Electrophysiological Imaging by. Ph.D. thesis, Rochester Insitute of Technology, 2015.

[43] Carpenter B. et.al. Stan: A Probabilistic Programming Language. J Stat Softw 2017;76(1). ISSN 1548-7660.

Address for correspondence:

Jaume Coll-Font - jaume.coll-font@ childrens.harvard.edu, Computational radiology Lab., Boston Children's Hospital

360 Longwood Ave., Boston, Massachusetts 02115 